\title{
Fab Four self-interaction in quantum regime
}

\author{
A. B. Arbuzov ${ }^{1,2, a}$, B. N. Latosh $h^{1,2, b}$ \\ ${ }^{1}$ Bogoliubov Laboratory for Theoretical Physics, JINR, Dubna 141980, Russia \\ 2 Dubna State University, Universitetskaya str. 19, Dubna, Moscow Region 141980, Russia
}

Received: 15 June 2017 / Accepted: 12 September 2017 / Published online: 24 October 2017

(C) The Author(s) 2017. This article is an open access publication

\begin{abstract}
Quantum behavior of the John Lagrangian from the Fab Four class of covariant Galileons is studied. We consider one-loop corrections to the John interaction due to cubic scalar field interaction. Counter terms are calculated, one appears because of massless scalar field theory infrared issues, another one lies in the George class, and the rest of them can be reduced to the initial Lagrangian up to surface terms. The role of quantum corrections in the context of cosmological applications is discussed.
\end{abstract}

\section{Introduction}

Scalar-tensor models of gravity belong to one of the simplest classes of modified gravity models, dating back to the BransDicke theory [1]. Such models arise in different contexts and possess a wide phenomenology. For instance, they appear in the context of low-energy string models [2], models with auxiliary dimensions [3], inflation [4] and wormholes [5,6]. Some scalar-tensor models can pass the Solar system tests because of the screening mechanism [7,8], which makes them even more attractive from the theoretical point of view.

In recent times a particular class of scalar-tensor models called Galileons has drawn a lot of attention; see [9] for a detailed review. This class was first discovered by Horndeski [10] who searched for the most general scalar-tensor model of gravity with second order field equations. Later it was shown that the Horndeski theory is equivalent to the theory of the so-called covariant Galileons [11]. Galileons originate from a low-dimensional reduction of braneworld models to a flat spacetime [12]. A compactification procedure spawns an additional scalar field with the so-called generalized Galilean symmetry which protects the differential order of field equations. Covariant Galileons are the most simple generalization of the Galileons for a curved space-

\footnotetext{
a e-mail: arbuzov@theor.jinr.ru

be-mail: latosh@gmail.com
}

time $[13,14]$. The generalization is performed by the extension of the Galileons Lagrangian with curvature-related terms that cancel out higher derivatives thus preserving the order of the field equations. This generalization comes with the price of the generalized Galilean symmetry, but it is possible to construct models that at least partially preserve the symmetry [15-17]. Covariant Galileons do not contain ghosts because of the order of the field equations, but it is possible to construct even more general ghost-free scalar-tensor model of gravity with higher derivatives [18]. Such models are known as beyond-Horndeski ones and they can be reduced to the Galileons by a proper redefinition of the dynamical variables.

In four dimensions the covariant Galileons are generated by the following Lagrangians:

$$
\begin{aligned}
\mathcal{L}_{2}= & G_{2} \\
\mathcal{L}_{3}= & -G_{3} \square \phi \\
\mathcal{L}_{4}= & G_{4} R+G_{4 X}\left[(\square \phi)^{2}-\left(\nabla_{\mu} \nabla_{\nu} \phi\right)^{2}\right] \\
\mathcal{L}_{5}= & G_{5} G_{\mu \nu} \nabla^{\mu} \nabla^{\nu} \phi-\frac{1}{6} G_{5 X}\left[(\square \phi)^{3}-3 \square \phi\left(\nabla_{\mu} \nabla_{\nu} \phi\right)^{2}\right. \\
& \left.+2\left(\nabla_{\mu} \nabla_{\nu} \phi\right)^{3}\right]
\end{aligned}
$$

where $G_{2}, G_{3}, G_{4}$, and $G_{5}$ are arbitrary functions of the Galileon field $\phi$ and the standard kinetic term $X=$ $1 / 2 \partial_{\mu} \phi \partial^{\mu} \phi$; the subscript $X$ denotes the derivative with respect to the standard kinetic term; $R$ is the Ricci curvature, and $G_{\mu \nu}$ is the Einstein tensor.

The covariant Galileons can be reduced to general relativity (GR) by setting $G_{2}=G_{3}=G_{5}=0$ and $G_{4}=1 / 16 \pi G$. At the same time, the cosmological constant should be included in the model as a free parameter [19] which is going to affect the cosmological properties of the covariant Galileons. In Ref. [20] it was shown that there exists a narrow subclass of the covariant Galileons which is able to screen the cosmological constant completely on the Friedmann-Lemaitre-Robertson-Walker background. This class is known as the Fab Four one; it is generated by the 
following Lagrangians:

$$
\begin{aligned}
L_{\text {John }} & =V_{J}(\phi) G^{\mu \nu} \nabla_{\mu} \phi \nabla_{\nu} \phi, \\
L_{\text {George }} & =V_{G}(\phi) R, \\
L_{\text {Ringo }} & =V_{R}(\phi) \hat{G}, \\
L_{\text {Paul }} & =V_{P}(\phi) P^{\mu \nu \alpha \beta} \nabla_{\mu} \phi \nabla_{\alpha} \phi \nabla_{\nu} \nabla_{\beta} \phi,
\end{aligned}
$$

where $\hat{G}$ is the Gauss-Bonnet term, $P^{\mu \nu \alpha \beta}=-1 / 2 \varepsilon^{\alpha \beta \lambda \tau}$ $R_{\lambda \tau \sigma \rho} \varepsilon^{\sigma \rho \mu v}$ is the double-dual Riemann tensor, and the functions $V_{J}, V_{G}, V_{P}, V_{R}$ are interaction potentials.

Thus the covariant Galileons provide an opportunity to extend GR in a way that might explain the smallness of the cosmological constant. One may take a Lagrangian from the Fab Four class and introduce some beyond-Fab Four terms. Such a model is going to screen the cosmological constant when Fab Four terms are dominant in the Lagrangian, so the model has room for a matter-dominated phase of the universe expansion. When beyond-Fab Four terms cannot be neglected, the model loses the ability to screen the cosmological constant and the universe enters the late-time acceleration phase. In Ref. [21] the simplest example of such a model is presented. The model includes the John Lagrangian with a constant potential $V_{J}$, the George Lagrangian with a constant potential $V_{G}=M_{\mathrm{Pl}}^{2}$ and the standard kinetic term for a scalar field (which belongs to $\mathcal{L}_{2}$ of the covariant Galileons). Following the aforementioned logic, the model provides a uniform description of inflation, a matter-dominated phase of expansion, and late-time acceleration of the universe.

The approach appears to be very fruitful, but it can be broken down at the quantum level, as was pointed out in the original paper [20]. In the realms of a flat spacetime the Galileons are protected from quantum corrections [22,23]; a similar situation might appear in the realm of curved spacetime. Counter terms associated with one-loop covariant Galileon corrections to the Fab Four interaction might not lie in the Fab Four class. In such a case quantum corrections might spawn a number of terms that cannot be neglected and are unable to screen the cosmological constant, thus ruining the desirable feature of the Fab Four class. An example of a similar behavior was recently found in the cubic covariant Galileons [24,25], within the gauge-invariant regularization scheme Galileons appears to receive additional higherderivative terms in one-loop effective Lagrangian.

In this paper we present a study of a simple model that contains both Fab Four and covariant Galileons interactions. We consider the John interaction because of the following. First, as was pointed out in Ref. [20] the Ringo term is unable to screen the cosmological constant, it just does not ruin the screening features of a model. Second, only the John and Paul terms provide an example of a non-minimal kinetic coupling that might posses some interesting properties, but the Paul term appears to demonstrate a pathological behavior in star-like objects $[26,27]$. Finally, as we pointed out before, the John Lagrangian provides a satisfactory description of the evolution of the universe in a similar model [21], which makes it the most perspective candidate for research. Following Ref. [21], we include the standard kinetic term for a scalar field in the model. As the additional beyond-Fab Four interaction that generates loop corrections we consider a standard $\phi^{3}$ interaction as it is the most simple scalar field self-interaction.

We consider scalar field cubic self-interaction because of the following reasons. First, the original model, considered in [21], contains no scalar field self-interaction, thus any quantum correction to the Fab Four interactions can appear only because of the virtual graviton exchange. Such corrections are suppressed by the square of the gravitational coupling and, despite having theoretical importance, can be neglected on practical grounds. In such a way, if one wants to consider the stability of the model with respect to quantum corrections, they need to introduce a scalar field self-interaction. Second, in the original paper [21] scalar field self-interaction is neglected because of the cosmological consideration, as it may violate the screening mechanism. But the model contains no symmetry forbidding scalar field self-interaction. Therefore, it appears to us, a model expanded with a minimal scalar field self-interaction appears to be more realistic.

This paper is organized as follows. In Sect. 2 we formulate the task and provide the solution to it. We show that corrections to the John interaction require counter terms that lie in the Fab Four class up to a surface turn. We discuss our results in Sect. 3. In the appendices we present the full set of Feynman rules for the model and a detailed discussion of infrared divergences of the model.

\section{Quantum corrections and renormalization}

As we mentioned before, we consider the simplest scalar field self-interaction $\phi^{3}$ along with the John interaction. We also include the standard kinetic term for the scalar field following Ref. [21]. In this way we consider the following set of Fab Four parameters:

$$
V_{R}=V_{P}=0, V_{J}=\beta_{0}+\beta_{1} \phi, V_{G}=\frac{1}{16 \pi G} .
$$

The case $\beta_{1}=0$ corresponds to the simplest situation, however, we consider $\beta_{1} \neq 0$. The cubic scalar field selfinteraction results in the interaction of a graviton with three scalar particles (the interaction of a graviton with a part of the matter stress-energy tensor associated with a scalar field potential); the John interaction with $\beta_{1} \neq 0$ also describes an interaction of a graviton with three scalar particles. Thus, these interactions interfere with each other and might bring about new divergences into the model, this is the reason for taking the case $\beta_{1} \neq 0$ into account. So, throughout the paper 
we consider the following action:

$$
\begin{aligned}
S= & \int \mathrm{d}^{4} x \sqrt{-g}\left[\frac{1}{16 \pi G} R+\frac{1}{2} \partial_{\mu} \phi \partial^{\mu} \phi+\frac{\lambda}{3 !} \phi^{3}\right. \\
& \left.+V_{J}(\phi) G^{\mu \nu} \partial_{\mu} \phi \partial_{\nu} \phi\right] .
\end{aligned}
$$

We are working within a low-energy regime and can treat the action (10) as an effective one. Following the standard linearization procedure within the harmonic gauge $\left(g^{\mu \nu} \Gamma_{\mu \nu}^{\lambda}=\right.$ 0 ) one obtains the following effective (linear) Lagrangian:

$$
\begin{aligned}
\mathcal{L}= & -\frac{1}{2} h^{\mu \nu} C_{\mu \nu \alpha \beta} \square h^{\alpha \beta}-\frac{1}{2} \phi \square \phi+\frac{\lambda}{3 !} \phi^{3} \\
& +\frac{\kappa}{2} \frac{\lambda}{3 !} \phi^{3} h-\frac{\kappa}{2} \partial_{\mu} \phi \partial_{\nu} \phi C^{\mu \nu \alpha \beta}(1 \\
& \left.+\beta_{0} \square+\phi \beta_{1} \square\right) h_{\alpha \beta},
\end{aligned}
$$

where $h_{\mu \nu}$ is a small metric perturbation, $\kappa^{2}=8 \pi G$ is the gravitational coupling and $C_{\mu \nu \alpha \beta}$ is defined as

$C_{\mu \nu \alpha \beta}=\eta_{\mu \alpha} \eta_{\nu \beta}+\eta_{\mu \beta} \eta_{\nu \alpha}-\eta_{\mu \nu} \eta_{\alpha \beta}$.

For the sake of simplicity we use the following terminology throughout the paper. We call $G^{\mu \nu} \partial_{\mu} \phi \partial_{\nu} \phi$ the standard John interaction, $G^{\mu \nu} \phi \partial_{\mu} \phi \partial_{\nu} \phi$ the cubic John interaction, and $\phi^{3}$ the cubic self-interaction. We present the expressions for the Feynman rules of the model in Fig. 4 in the appendices.

At the first order of the perturbation theory beyond the tree level one should consider only three one-loop amplitudes that affect the standard John interaction; the corresponding diagrams are presented in Figs. 1, 2, and 3. We present the detailed expressions for the amplitudes for Fig. 5 in the appendices, while here we discuss their properties. In the diagrams, the dotted vertices mark the interactions from the John class. Amplitudes for Figs. 1 and 2 contain infrared divergences which are canceled out by soft-particle radiation processes. These divergences are generated by the cubic self-interaction, they are not related to the John interaction and can be treated easily. As a matter of fact, these divergences are very similar to infrared divergences in quantum electrodynamics (we provide a more detailed discussion in Appendix B). We also would like to underline that there are two types of interactions mixed in the calculation: the standard GR interaction and the Fab Four one. We discuss the corresponding counter terms separately as they play different roles in the task.

The amplitude corresponding to Fig. 2 describes a correction related to the scalar field self-energy and this correction by no means is related to the Fab Four. This correction alone can be excluded from the consideration by a proper (re)definition of the Galileon field asymptotic states. Without the John interaction the expression can be renormalized by the standard kinetic counter term for the scalar field. When the John interaction is taken into account the expression also requires the John-like counter term for renormalization. In

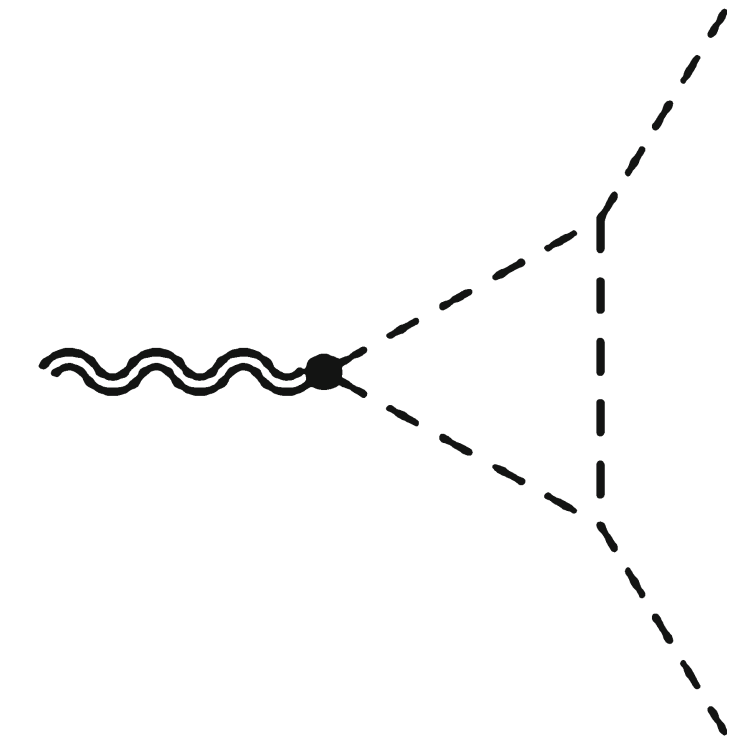

Fig. 1 Diagram for the first one-loop amplitude

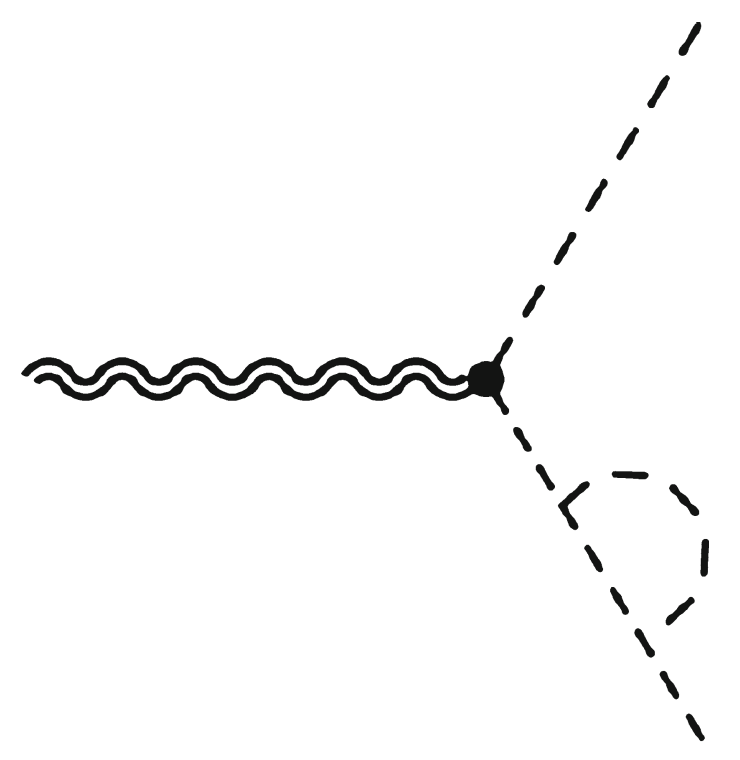

Fig. 2 Diagram for the second one-loop amplitude

such a way, the first amplitude can be renormalized completely within the original Lagrangian.

In the amplitude corresponding to Fig. 1 , only $R_{3}$ term (see Appendix A for notations) contains ultraviolet divergences that require renormalization. Without the John interaction the amplitude requires only the standard scalar field masslike counter term to be renormalized. This result should be understood as follows. First, without the John interaction the process contributes to renormalization of the matter stressenergy tensor, so it is not strongly related to the gravitational interaction, rather to the behavior of a massless scalar field. Second, it is well known that a quantum massless scalar field requires the introduction of a mass scale in the realms of flat 


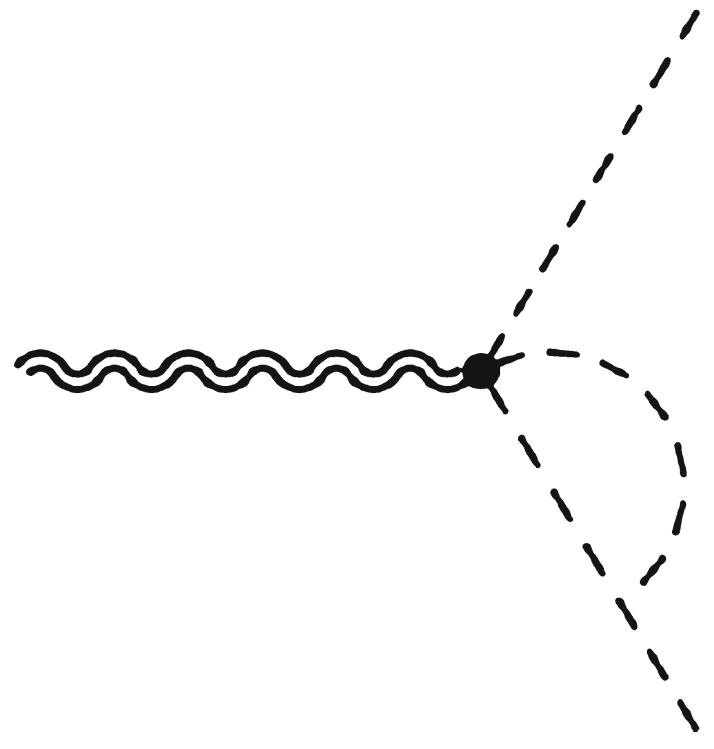

Fig. 3 Diagram for the third one-loop amplitude

spacetime [28]. So we treat the appearance of a mass-like counter term as a standard feature of a massless scalar field that is related neither with gravity nor with the Fab Four class. We do not discuss this feature of the model below as it is not related with the subject of our research and appears to be an inalienable property of a massless scalar field.

When the John interaction is taken into account the expression corresponding to Fig. 1 also requires the following counter term:

$C_{1}=R \phi^{2}$,

where $R$ is the scalar curvature. The detailed expression for the diagram in Fig. 1 is given in Fig. 5 (see (19), (20) and (21) for notation). The expression contains both infrared divergences (given by the factors $R_{1}$ and $R_{2}$ ) and ultraviolet divergences (given by the factor $R_{3}$ ). The regularization of infrared divergences is discussed in Appendix B, while the ultraviolet one results in the counter term (13). This counter term belongs to the George class of the Fab Four. Our model already contains one term from the George class, namely the standard GR Lagrangian which is generated by $V_{G}=1 / 16 \pi G$. Thus, our result indicates that one has to expand the George potential up to the second order terms:

$V_{G}=\frac{1}{16 \pi G}+\alpha \phi^{2}$,

where $\alpha$ is a new coupling. The appearance of (13) does not change the desirable features of the model, it rather points to a deep physical connection between the John and George classes.

The expression corresponding to Fig. 3 without the John interaction requires only the standard scalar field mass-like counter term. The situation is identical to the previous case and the same logic is applied. When the John interaction enters the consideration, the amplitude requires three more counter terms. The interaction of a graviton with three scalar particles belongs to the John class and has a structure similar to the standard John interaction. However, because of the difference in the structure of interactions, the corresponding counter terms can be reduced to the standard John (counter) term up to the surface term,

$C_{2}=\partial_{\mu}\left(\sqrt{-g} G^{\mu \nu} \phi \nabla_{\nu} \phi\right)$.

In the context of our task the term may be neglected, but it may play an important role in a cosmological task. Thus we showed that the Standard John interaction appears to be renormalizable up to the surface turn.

\section{Discussion and conclusion}

In the paper we showed that the simplest quantum correction to the John Lagrangian of the Fab Four class appears to be renormalizable. We considered the John Lagrangian as it provides the simplest example of scalar-tensor gravity with second order field equations and with the ability to screen the cosmological constant. The model is fruitful as it provides a uniform description of the evolution of the universe from inflation till late-time accelerated expansion [21]. The model has this feature because of the ability to screen the cosmological constant at a certain epoch of the evolution providing room to the matter-dominated phase of the expansion. In such a way the ability to screen the cosmological constant becomes crucial for the cosmological applications of the model.

At the same time, the model is not protected from quantum corrections and they might ruin that desirable feature. We studied the simplest case of such quantum corrections coming from the standard cubic scalar field self-interaction. Our results show that such corrections require the introduction of one counter term (13) that does not belong to the initial Lagrangian.

The counter term (13) belongs to the Fab Four class, thus it cannot ruin the desirable property of the model. Moreover, (13) belongs to the George class, which is already present in the model; thus it only indicates the necessity to take into account higher order terms of the George potential expansion into account:

$$
V_{G}=\frac{1}{16 \pi G}+\alpha \phi^{2} .
$$

This result shows that the John and George classes have a non-trivial physical connection and the standard John interaction requires the presence of the George interaction for renormalization. 
Because of the massless scalar field infrared issues [28] the model requires the standard mass-like counter term for renormalization. However, the issue is related with the properties of the scalar field and by no means related with gravity; therefore we did not discuss this issue of the model. The rest of the counter terms can be reduced to the standard John Lagrangian up to the surface term (15).

Summarizing all of the above, we demonstrated that the John and George classes of the Fab Four have a physical connection through the standard scalar field self-interaction. A proper choice of the George potential may lead to the renormalizability of the model at the level of one-loop amplitudes.

To present a more detailed judgment as regards the stability of the particular model, and as regards the Fab Four in general, one still needs to go beyond three-particle amplitudes. However, this task is much more sophisticated and lies beyond the scope of this article. The task is complicated by two issues. First, if one considers high multiplicity amplitudes, that would require one to introduce multi-particle interactions in the model. In the realm of flat spacetime such interactions may contribute beyond Galileon counter terms $[22,23]$. That phenomenon requires a special treatment, which goes beyond this study. Second, the consideration of high multiplicity amplitudes requires the introduction of multi-particle interaction terms in the model. In this case the number of diagrams required for the calculation increases rapidly and the task becomes overcomplicated from the computational point of view.

Acknowledgements This work was supported by Russian Foundation for Basic Research via Grant RFBR 16-02-00682. The authors are also grateful to Antonio Padilla for a very useful comment on surface terms.

Open Access This article is distributed under the terms of the Creative Commons Attribution 4.0 International License (http://creativecomm ons.org/licenses/by/4.0/), which permits unrestricted use, distribution, and reproduction in any medium, provided you give appropriate credit to the original author(s) and the source, provide a link to the Creative Commons license, and indicate if changes were made.

Funded by SCOAP ${ }^{3}$.

\section{Appendix A. Feynman rules and one-loop amplitudes}

Quantities used in the article have the following mass dimensions: $[\phi]=[\lambda]=1,[\kappa]=-1,\left[\beta_{0}\right]=-2,\left[\beta_{1}\right]=-3$. Feynman rules are given in Fig. 4. In all diagrams the momenta are pointed right and the following definitions for $C$ and $D$-symbols are used [29]:

$$
\begin{aligned}
C_{\mu \nu \alpha \beta}= & \eta_{\mu \alpha} \eta_{\nu \beta}+\eta_{\mu \beta} \eta_{\nu \alpha}-\eta_{\mu \nu} \eta_{\alpha \beta}, \\
D_{\mu \nu \alpha \beta}\left(k_{1}, k_{2}\right)= & \eta_{\mu \nu} k_{1 \alpha} k_{2 \beta}-\left[\eta_{\mu \alpha} k_{1 \nu} k_{2 \beta}+\eta_{\mu \beta} k_{1 \alpha} k_{2 v}\right. \\
& \left.-\eta_{\alpha \beta} k_{1 \mu} k_{2 v}+(\mu \leftrightarrow \nu)\right] .
\end{aligned}
$$

Expressions for one-loop amplitudes discussed in the paper are presented in Fig. 5 and the following set of definitions is used: $\int \frac{\mathrm{d}^{4} k}{(2 \pi)^{4}} \frac{1}{k^{2}\left(k-p_{1}\right)^{2}\left(k+p_{2}\right)^{2}}=\frac{i}{16 \pi^{2}} \frac{1}{\left(p_{1}+p_{2}\right)^{2}} R_{1}$,

$\int \frac{\mathrm{d}^{4} k}{(2 \pi)^{4}} \frac{k_{\mu}}{k^{2}\left(k-p_{1}\right)^{2}\left(k+p_{2}\right)^{2}}=\frac{i}{16 \pi^{2}} \frac{\left(p_{1}-p_{2}\right)_{\mu}}{\left(p_{1}+p_{2}\right)^{2}} R_{2}$,

$\int \frac{\mathrm{d}^{4} k}{(2 \pi)^{4}} \frac{C_{\mu \nu \alpha \beta} k^{\alpha} k^{\beta}}{k^{2}\left(k-p_{1}\right)^{2}\left(k+p_{2}\right)^{2}}=-\frac{1}{2} \eta_{\mu \nu} \frac{i}{16 \pi^{2}} R_{3}$,

$\int \frac{\mathrm{d}^{4} k}{(2 \pi)^{4}} \frac{1}{k^{2}(k+p)^{2}}=\frac{i}{16 \pi^{2}} R_{4}$,

$\int \frac{\mathrm{d}^{4} k}{(2 \pi)^{4}} \frac{k_{\mu}}{k^{2}(k+p)^{2}}=-\frac{1}{2} p_{\mu} \frac{i}{16 \pi^{2}} R_{4}$,

$R_{1}=\frac{\Gamma(1-\varepsilon) \Gamma^{2}(\varepsilon)}{2 \varepsilon \Gamma(2 \varepsilon)}\left(-\frac{1}{4 \pi} \frac{\left(p_{1}+p_{2}\right)^{2}}{\mu^{2}}\right)^{\varepsilon}$,

$R_{2}=\frac{\Gamma(1-\varepsilon) \Gamma(\varepsilon) \Gamma(1+\varepsilon)}{(1+2 \varepsilon) \Gamma(1+2 \varepsilon)}\left(-\frac{1}{4 \pi} \frac{\left(p_{1}+p_{2}\right)^{2}}{\mu^{2}}\right)^{\varepsilon}$,

$R_{3}=\frac{\Gamma(\varepsilon) \Gamma^{2}(1-\varepsilon)}{\Gamma(2-\varepsilon)}\left(-\frac{1}{4 \pi} \frac{\left(p_{1}+p_{2}\right)^{2}}{\mu^{2}}\right)^{\varepsilon}$,

$R_{4}=\frac{1}{\varepsilon \Gamma(2+\varepsilon)}\left(\frac{1}{4 \pi} \frac{\Lambda^{2}}{\mu^{2}}\right)^{\varepsilon}$.

The factors $R_{1}$ and $R_{2}$ contain infrared-divergent parts of the amplitudes, while $R_{3}$ and $R_{4}$ contain ultraviolet ones. The parameter $\Lambda$ is used as the ultraviolet cut-off.

\section{Appendix B. Infrared stability}

The amplitude corresponding to Fig. 1 contains the following infrared-divergent part:

$$
-\frac{\kappa}{2}\left(1-\beta_{0} q^{2}\right) \lambda^{2} C^{\mu \nu \alpha \beta}\left(p_{1}\right)_{\alpha}\left(p_{2}\right)_{\beta} \int \frac{\mathrm{d}^{4} k}{(2 \pi)^{4}} \frac{1}{k^{2}\left(k-p_{1}\right)^{2}\left(k-p_{2}\right)^{2}} .
$$

In this expression integration over $k^{0}$ should be performed:

$$
\begin{aligned}
& \int \frac{\mathrm{d}^{4} k}{(2 \pi)^{4}} \frac{1}{k^{2}\left(k-p_{1}\right)^{2}\left(k-p_{2}\right)^{2}} \\
& =\left.\frac{i}{8} \int \frac{\mathrm{d}^{3} \mathbf{k}}{(2 \pi)^{3}} \frac{1}{k^{0}} \frac{1}{k \cdot p_{1} k \cdot p_{2}}\right|_{k^{2}=0} \\
& \quad-\frac{i}{8} \int \frac{\mathrm{d}^{3} \mathbf{k}}{(2 \pi)^{3}} \frac{1}{k^{0}}\left\{\frac{1}{\left(k \cdot p_{1}\right)\left(k \cdot\left(p_{1}-p_{2}\right)+p_{1} \cdot p_{2}\right)}\right. \\
& \left.+\left[p_{1} \leftrightarrow p_{2}\right]\right\}\left.\right|_{k^{2}=0} .
\end{aligned}
$$

In (29) only the first term diverges in the infrared sector. The divergent integral is the standard one that appears in soft- 


$$
------=\frac{i}{k^{2}}
$$

$\mu \nu \approx \sim \approx \Omega\left(\alpha \beta=i \frac{C_{\mu \nu \alpha \beta}}{k^{2}}\right.$

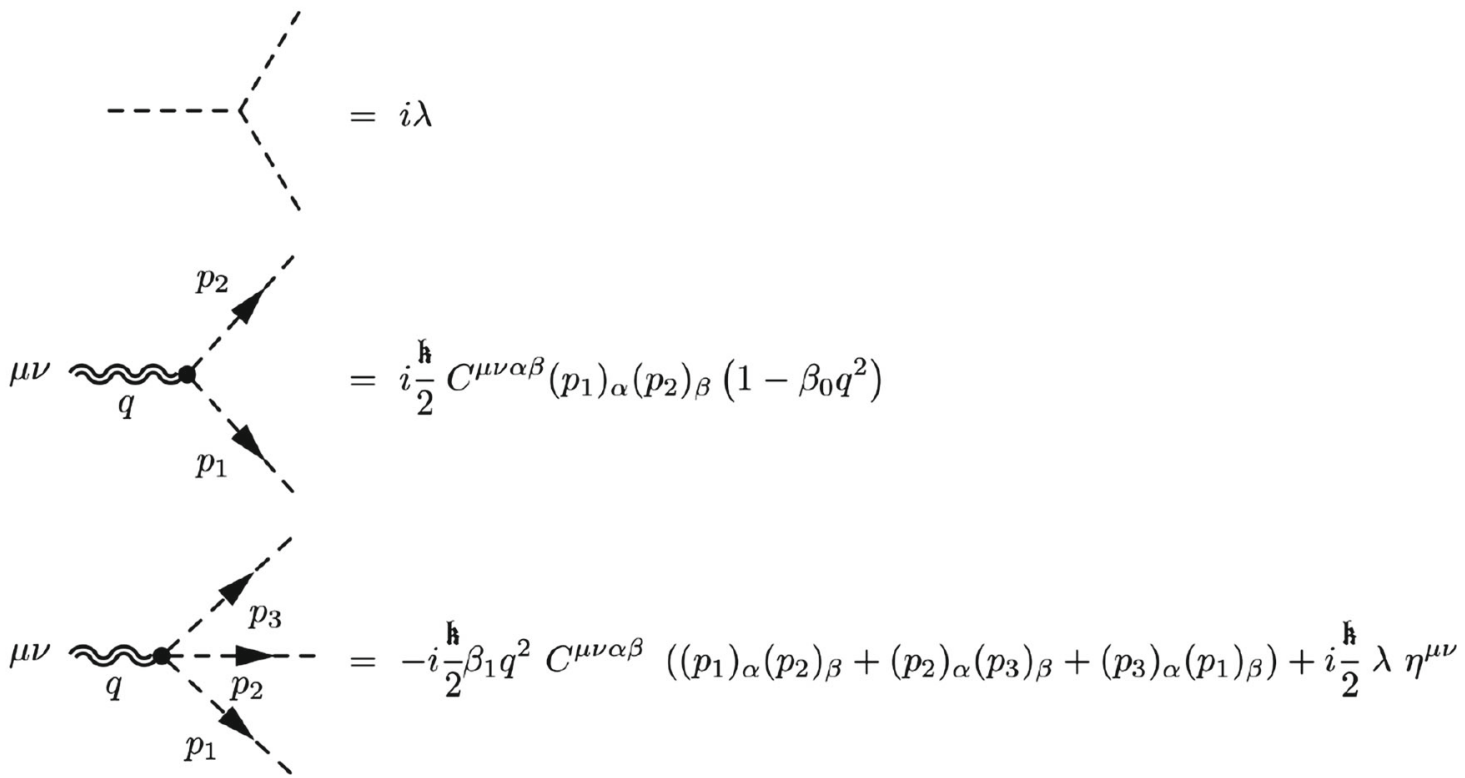

Fig. 4 Feynman rules for the model

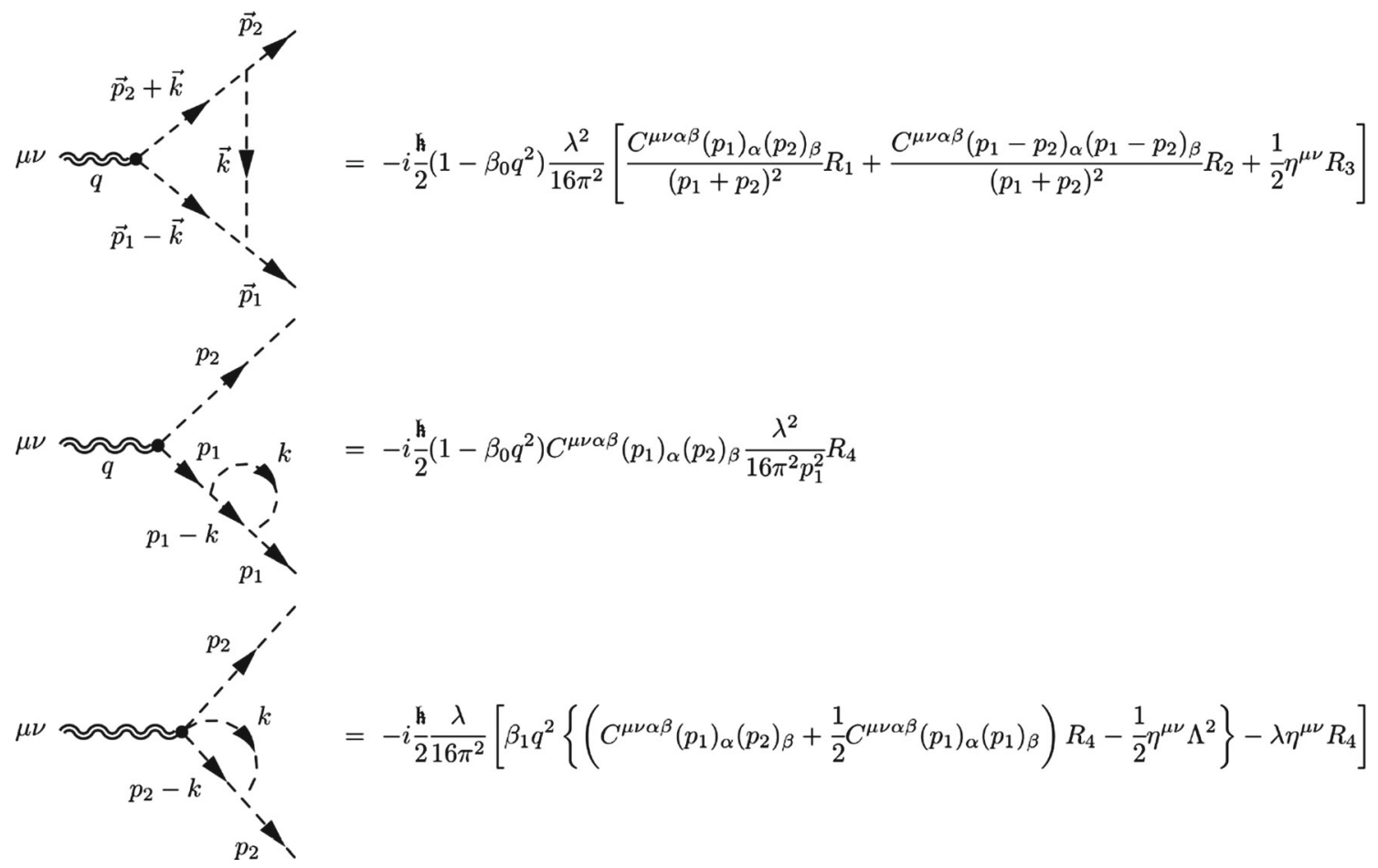

Fig. 5 Expressions for one-loop amplitudes 
particle radiation amplitudes, therefore the divergent part of the amplitude is regularized by soft-particle radiation.

The same logic holds for the amplitude corresponding to Fig. 2. The divergent part of the expression is given by the following:

$i \frac{\kappa}{2}\left(1-\beta_{0} q^{2}\right) C^{\mu \nu \alpha \beta}\left(p_{1}\right)_{\alpha}\left(p_{2}\right)_{\beta} i \frac{\lambda^{2}}{p_{1}^{2}} \int \frac{\mathrm{d}^{4} k}{(2 \pi)^{4}} \frac{1}{k^{2}\left(k-p_{1}\right)^{2}}$.

In a similar way integration over $k^{0}$ can be performed:

$\frac{1}{p_{1}^{2}} \int \frac{\mathrm{d}^{4} k}{(2 \pi)^{4}} \frac{1}{k^{2}\left(k-p_{1}\right)^{2}}=-\left.\frac{i}{4} \int \frac{\mathrm{d}^{3} \mathbf{k}}{(2 \pi)^{3}} \frac{1}{k^{0}} \frac{1}{\left(k \cdot p_{1}\right)^{2}}\right|_{k^{2}=0}$.

In full analogy with the previous result we obtain the standard integral for the soft-particle radiation amplitude. At the same time, Eq. (31) looks as if a factor $1 / 2$ is missing, but this is not the case. All scalar particles in our theory are identical, thus additional permutation of outgoing particles is necessary. As a consequence, the corresponding soft-particle radiation amplitude receives the additional factor 2 and exactly compensates by the infrared-divergent term.

\section{References}

1. C. Brans, R.H. Dicke, Mach's principle and a relativistic theory of gravitation. Phys. Rev. 124, 925-935 (1961)

2. D.J. Gross, J.H. Sloan, The quartic effective action for the heterotic string. Nucl. Phys. B 291, 41-89 (1987)

3. J.E. Kim, B. Kyae, H.M. Lee, Effective Gauss-Bonnet interaction in Randall-Sundrum compactification. Phys. Rev. D 62, 045013 (2000)

4. A.D. Linde, Chaotic inflation. Phys. Lett. B129, 177-181 (1983)

5. T.A. Roman, Inflating Lorentzian wormholes. Phys. Rev. D 47, 1370-1379 (1993)

6. M. Jamil, P.K.F. Kuhfittig, F. Rahaman, S.A. Rakib, Wormholes supported by polytropic phantom energy. Eur. Phys. J. C67, 513$520(2010)$

7. A.I. Vainshtein, To the problem of nonvanishing gravitation mass. Phys. Lett. B 39, 393-394 (1972)

8. E. Babichev, C. Deffayet, An introduction to the Vainshtein mechanism. Class. Quantum Gravity 30, 184001 (2013)
9. C. Deffayet, D.A. Steer, A formal introduction to Horndeski and Galileon theories and their generalizations. Class. Quantum Gravity 30, 214006 (2013)

10. G.W. Horndeski, Second-order scalar-tensor field equations in a four-dimensional space. Int. J. Theor. Phys. 10, 363-384 (1974)

11. T. Kobayashi, M. Yamaguchi, J. Yokoyama, Generalized Ginflation: inflation with the most general second-order field equations. Prog. Theor. Phys. 126, 511-529 (2011)

12. A. Nicolis, R. Rattazzi, E. Trincherini, The Galileon as a local modification of gravity. Phys. Rev. D 79, 064036 (2009)

13. C. Deffayet, G. Esposito-Farese, A. Vikman, Covariant Galileon. Phys. Rev. D 79, 084003 (2009)

14. C. Deffayet, S. Deser, G. Esposito-Farese, Generalized Galileons: all scalar models whose curved background extensions maintain second-order field equations and stress-tensors. Phys. Rev. D 80, 064015 (2009)

15. D. Pirtskhalava, L. Santoni, E. Trincherini, F. Vernizzi, Weakly broken Galileon symmetry. JCAP 1509(09), 007 (2015)

16. C. Germani, L. Martucci, P. Moyassari, Introducing the Slotheon: a slow Galileon scalar field in curved space-time. Phys. Rev. D 85, 103501 (2012)

17. R. Banerjee, S. Chakraborty, A. Mitra, P. Mukherjee, Cosmological implications of shift symmetric Galileon field. Phys. Rev. D96 (6) 064023 (2017)

18. J. Gleyzes, D. Langlois, F. Piazza, F. Vernizzi, Healthy theories beyond Horndeski. Phys. Rev. Lett. 114(21), 211101 (2015)

19. D. Lovelock, The Einstein tensor and its generalizations. J. Math. Phys. 12, 498-501 (1971)

20. C. Charmousis, E.J. Copeland, A. Padilla, P.M. Saffin, General second order scalar-tensor theory, self tuning, and the Fab Four. Phys. Rev. Lett. 108, 051101 (2012)

21. A.A. Starobinsky, S.V. Sushkov, M.S. Volkov, The screening Horndeski cosmologies. JCAP 1606(06), 007 (2016)

22. M.A. Luty, M. Porrati, R. Rattazzi, Strong interactions and stability in the DGP model. JHEP 09, 029 (2003)

23. K. Hinterbichler, M. Trodden, D. Wesley, Multi-field Galileons and higher co-dimension branes. Phys. Rev. D 82, 124018 (2010)

24. I.D. Saltas, V. Vitagliano, Quantum corrections for the cubic Galileon in the covariant language. JCAP 1705(05), 020 (2017)

25. I.D. Saltas, V. Vitagliano, Covariantly quantum Galileon. Phys. Rev. D 95(10), 105002 (2017)

26. S. Appleby, Self tuning scalar fields in spherically symmetric spacetimes. JCAP 1505, 009 (2015)

27. A. Maselli, H.O. Silva, M. Minamitsuji, E. Berti, Neutron stars in Horndeski gravity. Phys. Rev. D 93(12), 124056 (2016)

28. S.R. Coleman, E.J. Weinberg, Radiative corrections as the origin of spontaneous symmetry breaking. Phys. Rev. D 7, 1888-1910 (1973)

29. T. Han, J.D. Lykken, R.-J. Zhang, On Kaluza-Klein states from large extra dimensions. Phys. Rev. D 59, 105006 (1999) 